\title{
Does Atelocollagen Actually Help the Rotator Cuff Healing and Pain Control?
}

\author{
Kyu-Hak Jung \\ Department of Orthopaedic Surgery, Gil Medical Center, Gachon University College of Medicine, Incheon, Korea
}

Collagen plays an important role in withstanding mechanical failure in connective tissue, and this property has attracted considerable attention for its relationship with cuff healing. ${ }^{1)}$ In particular, atelocollagen is a highly purified collagen with low immunogenicity and is used widely because of its good biocompatibility. ${ }^{2)}$ On the other hand, there are few studies on cuff healing. In "Does the use of injectable atelocollagen during arthroscopic rotator cuff repair improve clinical and structural outcomes?" (Clin Shoulder Elbow 2019;22(4):183-189), Kim et al. compared the results of using gel-type atelocollagen on the rotator cuff repair for an average follow-up of 26.7 months. At the last follow-up, there was no difference in cuff healing compared to the group with and without atelocollagen. Interestingly, the group that used atelocollagen showed significant improvement in short-term pain within two weeks after surgery, but there was no difference between the two groups in the clinical scores at three, 12, and 24 months.

In a recent study using atelocollagen, Suh et al. ${ }^{3)}$ suggested repair after using patch-type atelocollagen between the torn rotator cuff and bone using rabbits. As a result, the group that used atelocollagen showed pathological and biomechanical superiority. Several differences between Suh et al.'s and Kim et al.'s data can be noted. ${ }^{3)}$ On the other hand, the most different thing is the type of atelocollagen (patch vs. gel). Several studies have shown the superiority of structural integrity after rotator cuff repair using patch-type collagen. On the other hand, Kim et al.'s research ${ }^{4)}$ was the only one using gel-type atelocollagen. In the study by Kim et al., ${ }^{4)}$ follow-up MRI confirmed the change in cuff integrity after injecting atelocollagen into the intratendinous tear site, and significant improvement was observed in the group with atelocollagen. On the other hand, the reason for the difference between Kim et al.'s research and Kim et al.'s research ${ }^{4)}$ is difficult to clarify because of the few studies published. Therefore, the overuse of the atelocollagen to improve cuff healing should be reduced.

The authors described the short-term improvement of the pain within two weeks after surgery because atelocollagen had an anti-inflammatory effect on the early tendon healing stage. In addition, the effect was different from that of the conventional injection into the subacromial space because collagen was injected between the repaired tendon and the bone. Magarian et al. ${ }^{5)}$ showed that there was no increase in inflammation, including the synovial fluid leukocytes and synovial thickening, in the group using atelocollagen after the anterior cruciate ligament repair. No direct study on atelocollagen that reduces inflammation has been reported. Nevertheless, considering that atelocollagen did not at least increase inflammation, further study will be needed to confirm the relationship among the atelocollagen, inflammation, and pain.

Atelocollagen is "hot" in the area of the rotator cuff, but many materials still need to be identified correctly. Kim et al. suggested several issues. Further research on various aspects of atelocollagen will be needed.

\section{References}

1. Pins GD, Christiansen DL, Patel R, Silver FH. Self-assembly of collagen fibers. Influence of fibrillar alignment and decorin on mechanical properties. Biophys J. 1997;73(4):2164-72. doi: 10.1016/S0006-3495(97)78247-X.

2. Lynn AK, Yannas IV, Bonfield W. Antigenicity and immunogenicity of collagen. J Biomed Mater Res B Appl Biomater. 2004;71(2):343-54. doi: 10.1002/jbm.b.30096.

3. Suh DS, Lee JK, Yoo JC, et al. Atelocollagen enhances the heal-

\section{Correspondence to: Kyu-Hak Jung}

Department of Orthopaedic Surgery, Gachon University Gil Medical Center, 21 Namdong-daero 774beon-gil, Namdong-gu, Incheon 21565, Korea

Tel: +82-32-460-3384, Fax: +82-32-423-3384, E-mail: jjangumom52@hanmail.net, ORCID: https://orcid.org/0000-0003-0211-8005

Editorial does not need an IRB approval.

Financial support: None. Conflict of interests: None. 
ing of rotator cuff tendon in rabbit model. Am J Sports Med. 2017;45(9):2019-27. doi: 10.1177/0363546517703336.

4. Kim YS, Lee HJ, Kim DJ, Seo JY, Jang G. The effect of atelocollagen injection in partial thickness rotator cuff tears: a prospective randommized clinical evaluation measured by repeated MRI. Paper presented at: The 26th Congress of Korean Shoul- der and Elbow Society; 2018 Mar 30; Seoul, Korea.

5. Magarian EM, Vavken P, Connolly SA, Mastrangelo AN, Murray $\mathrm{MM}$. Safety of intra-articular use of atelocollagen for enhanced tissue repair. Open Orthop J. 2012;6:231-8. doi: 10.2174/1874325001206010231. 